\title{
SERUM IRISIN LEVEL INCREASES THROUGHOUT THE GESTATIONAL PERIOD AND IT DOES NOT PLAY A ROLE IN DEVELOPMENT OF GESTATIONAL DIABETES MELLITUS
}

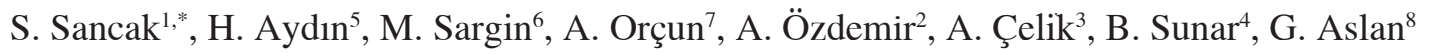 \\ Fatih Sultan Mehmet Training and Educational Hospital, ${ }^{1}$ Division of Endocrinology and Metabolism, ${ }^{2}$ Dept. \\ of Internal Medicine, ${ }^{3}$ Division of Obstetrics and Gynecology, ${ }^{4}$ Division of Biochemistry, Yeditepe University \\ Hospital, ${ }^{5}$ Dept. of Endocrinology and Metabolism, Kartal Lutfi Kirdar Training and Educational Hospital, \\ ${ }^{6}$ Diabetes Department, ${ }^{7}$ Division of Biochemistry, ${ }^{8}$ Dept. of Internal Medicine, Istanbul, Turkey
}

\begin{abstract}
Introduction. Irisin is a recently discovered novel adipomyokine that induces an increase in total body energy expenditure, improves insulin sensitivity and glucose tolerance. It has been shown that circulating levels of irisin are low in patients with obesity, diabetes mellitus and impaired glucose tolerance. However, the information about the level of circulating irisin in gestational diabetes mellitus (GDM) is controversial.

Material and Methods. Serum irisin was measured by an ELISA in a longitudinal prospective cohort study in 221 women. There were 156 healthy pregnant and 65 women with GDM.

Results. Circulating irisin levels were significantlly higher in the middle pregnancy compared with early pregnancy levels in healthy pregnant women and in women with GDM. Serum irisin levels were found to be lower in GDM compared to healthy pregnant women during first trimester but the difference was not observed throughout the pregnancy and it was comparable in middle pregnancy. There was a significant inverse correlation of BMI with serum irisin $(r=-0.193, p=$ 0.004) and between HbA1c and mean glucose of OGTT with serum irisin $(r=-0.377, p=0.0001)$ and $(r=-0.147, p: 0.03)$ in the early pregnancy of pregnant women repectively.

Conclusions. The present study shows that serum irisin level increases throughout the gestational period from early to middle pregnancy in women with GDM, but there is no effect of irisin on the development of GDM.
\end{abstract}

Key words: Irisin, gestational diabetes mellitus, myokine, insuline resitance.

\section{INTRODUCTION}

Irisin is a novel discovered myokine, which is encoded by the fibronectin type III domain-containing protein 5 (FNDC5) precursor gene and regulates the differentiation and phenotype of adipose tissue (1-3).
Irisin drives brown-fat-like conversion of white adipose tissue $(3,4)$, thereby increasing the energy expenditure and reducing weight and insulin resistance (3, 5). Thus, these data suggest that a decrease in circulating irisin may be associated with the development of insulin resistance and impaired glucose tolerance.

GDM has been defined as any degree of glucose intolerance with onset or first recognition during pregnancy (6) or is regarded to be a transient unmasking of the metabolic syndrome, characterized by an enormous insulin resistance and inadequate betacell compensation (7). Despite extensive research, the mechanisms underlying insulin resistance are not fully understood.

GDM threatens both the mother and fetus. Thus, GDM patients have an increased risk of comorbidities during pregnancy, e.g. preeclampsia, pregnancy-induced hypertension, and shoulder dystocia with impeded delivery (8).

Furthermore, chronic complications might occur after delivery including type 2 diabetes mellitus (T2DM), obesity and cardiovascular disease in both the mother and offspring (9-11).

Different myokines and adipomyokines have been studied throughout gestation and have been proposed as biomarkers of GDM (12).

Recent studies have shown that irisin levels are low in patients with type 2 diabetes compared with nondiabetics $(4,13)$ and, in morbidly obese patients the circulating levels of irisin are significantly elevated when compared with normal-weight subjects $(2,14)$. A decrease in circulating irisin was also observed in patients with GDM $(15,16)$. However, the information of the levels of circulating irisin in GDM are controversial since two studies did not show significant differences between subjects with GDM

*Correspondence to: Seda Sancak, Erenköy, Istanbul, 34070, Turkey, E-mail: drsedasancak@gmail.com

Acta Endocrinologica (Buc), vol. XIII, no. 4, p. 393-399, 2017 
and healthy pregnant women $(17,18)$. There are few studies reporting increased levels of irisin during the gestational period (19), in which serum levels of irisin are higher during the entire pregnancy when compared with nonpregnant women (19).

To date there are few studies of the levels of irisin during the gestational period including GDM patients, which analyzed serum irisin levels in middle pregnancy in women with $\operatorname{GDM}(15,16,18)$.

The purpose of this study was to determine: a) the levels of irisin over the early and middle pregnancy b) the change of irisin between early and middle pregnancy in GDM subjects as a prospective and novel marker for GDM.

\section{MATERIALS AND METHODS}

This prospective study was conducted in the Department of Endocrinology and Metabolism and Department of Obstetrics and Gynecology. It was approved by the ethical committee (Date: 27/12/2012; Decision Number: FSM EAH-KAEK 2012/25) and written informed consent was obtained from all subjects. The study was a non-matched case-control study nested in a longitudinal cohort study.

Patients with severe conditions including multiple pregnancy, generalized or other chronic inflammation, vascular disease, chronic hypertension, acute or chronic renal or liver disease, neurological disorders, polycystic ovary syndrome, immunosuppressive treatment, current or recent use of systemic high-dose corticosteroids, use of beta-blockers, and other drugs that could affect the metabolism, preexisting diabetes, malignancy, chronic alcohol consumption and history of smoking were excluded from the study.

Women were included between the 11th and 13th week of gestation as determined by early ultrasound and last menstrual period. All the pregnant women were studied during early $\left(11^{\text {th }}-13^{\text {th }}\right.$ week of gestation) and middle pregnancy $\left(24^{\text {th }}-28^{\text {th }}\right.$ week of gestation), between 2012-2014.

Two hundred twenty one consecutive pregnant women were recruited for the study. Of these 65 women were diagnosed as GDM and 156 were healthy pregnant women.

GDM was diagnosed based on World Health Organization criteria following a 75-g oral glucose tolerance test at 24-28 week of gestation $(6,20)$.

\section{Laboratory assays}

Whole blood was drawn into 5.0-mL BD Vacutainer serum tubes from a vein of the upper arm. The blood sample was left at room temperature for 20 minutes, and then the clotted blood was centrifuged at $3000 \times \mathrm{g}$ for 10 minutes at $4^{\circ} \mathrm{C}$. Serum samples were immediately transferred and stored in separate tubes. Serums were stored at $-80^{\circ} \mathrm{C}$ until the biochemical and hormonal analyses were performed; any freezethaw cycles were avoided. Insulin measurements were performed by electrochemiluminescence immunoassay (ECLIA) on Cobas analyser (Roche, Germany) and glucose was measured by a hexokinase method on AU 5800 analyser (Beckman Coulter, USA). Glycated hemoglobin(HbA1c) was evaluated by a high performance liquid chromatography technique ( BIORAD Laboratories). Homeostasis model assessment of estimated insulin resistance (HOMA-IR) was determined using the formula described by Matthews et al. (21): HOMA = fasting insulin (microunits per milliliter $) \times$ fasting glucose (millimoles per liter)/ 22.5) .

Blood sampling was performed together with early pregnancy (between the $11^{\text {th }}$ and $13^{\text {th }}$ week of gestation) and $0 \mathrm{~h}$ OGTT time point after an overnight fast.

\section{Determination of human irisin in the serum}

Serum irisin levels were determined using a commercially available ELISA (BioVendor Research and Diagnostic Products, cat no: RAG018R). The assay range of irisin was $0.001-5 \mu \mathrm{g} / \mathrm{mL}$. The intraassay CV was $7.63 \%$ for $1.37 \mu \mathrm{g} / \mathrm{mL}$; interassay CV was $8.02 \%$ for $1.14 \mu \mathrm{g} / \mathrm{mL}$.

\section{Statistical analysis}

The data were analyzed using SPSS 20.0 software. Data was normally distributed and reported as mean \pm SD. Statistical significance was determined by the student $\mathrm{t}$ test for repeated measures. We performed a univariate analysis to examine the correlation between the irisin serum levels and biochemical and anthropometric variables in each trimester independently, using a Pearson's correlation coefficient. Finally, a multivariate linear regression model was carried out to estimate the effect of irisin on HOMA-IR, adjusting for the following variables: body mass index (BMI), trimester of gestation. For this model, the dependent variable HOMA-IR was normalized to its natural logarithm given its distribution. A value of $\mathrm{p}<0.01$ was considered statistically significant. 


\section{RESULTS}

Demographic and biochemical characteristics

Demographic and biochemical parameters and irisin levels of the subgroups studied and summarized in Tables 1 and 2.

\section{Baseline characteristics}

The women with GDM were older than healthy pregnant women $(31 \pm 5$ vs $28 \pm 5$; $\mathrm{p}<0.0001)$.

The women with GDM were significantly heavier than healthy pregnant women both in early and middle pregnancy $(71.60 \pm 14.58 \mathrm{~kg}$ vs $65.11 \pm 11.99 \mathrm{~kg}$, p: 0.0007$)$ and $(77.43 \pm 14.24 \mathrm{~kg}$ vs $72.56 \pm 13.05 \mathrm{~kg}$, p:0.0003) respectively.

The women with GDM had significantly higher BMI than healthy pregnant women in early and middle pregnancy $\left(28.24 \pm 5.41 \mathrm{~kg} / \mathrm{m}^{2}\right.$ vs $\left.25.29 \pm 4.35 \mathrm{~kg} / \mathrm{m}^{2}\right)$ and $\left(30.55 \pm 5.21 \mathrm{~kg} / \mathrm{m}^{2}\right.$ vs $\left.27.39 \pm 4.3 \mathrm{~kg} / \mathrm{m}^{2}\right)$, respectively. In addition, there was no significant difference between gestational diabetic pregnant women as compared with healthy pregnant women in the amount of weight gained during pregnancy $(5.83 \pm 4.73 \mathrm{~kg} v s 5.42 \pm 3.37 \mathrm{~kg}$, p:ns).
The patients with GDM had significantly higher fasting glucose and higher insulin than the healthy pregnant women in early pregnancy $(88.80 \pm 8.57 \mathrm{mg} /$ $\mathrm{dL} v s 83.39 \pm 6.89 \mathrm{mg} / \mathrm{dL}, \mathrm{p}<0.0001)$ and $(9.94 \pm 5.68$ $\mathrm{uU} / \mathrm{mL}$ vs $7.2 \pm 3.67 \mathrm{uU} / \mathrm{mL}, \mathrm{p}<0.0001$ ) respectively.

\section{Serum irisin levels of women diagnosed with gestational diabetes and normal controls}

Irisin levels were higher in the middle pregnancy compared with early pregnancy levels, with an increase in levels of about $61 \%(1.32 \pm 0.76 \mu \mathrm{g} / \mathrm{mL}$ vs $0.7567 \pm 0.54 \mu \mathrm{g} / \mathrm{mL}$, respectively, $\mathrm{p}<0.0001)$ in all pregnant women.

On the other hand, serum irisin levels were higher in middle pregnancy with respect to early pregnancy in healthy pregnant women and in women with GDM $(1.34 \pm 0.70 \mu \mathrm{g} / \mathrm{mL}$ vs $0.81 \pm 0.6 \mu \mathrm{g} / \mathrm{mL}$; $\mathrm{p}<0.0001)$ and $(1.28 \pm 0.89 \mu \mathrm{g} / \mathrm{mL}$ vs $0.62 \pm 0.36 \mu \mathrm{g} / \mathrm{mL}$, $\mathrm{p}<0.0001$ ) respectively (Fig. 1).

In addition, in the serum irisin levels obtained, there was a significant difference in gestational pregnant women as compared with healthy pregnant women in early $(0.6202 \pm 0.35678 \mu \mathrm{g} / \mathrm{mL} v s \quad 0.8136 \pm 0.5961 \mu \mathrm{g} /$

Table 1. Demographic and biochemical parameters and irisin levels of the healthy and gestational diabetic women

\begin{tabular}{|c|c|c|c|c|c|c|}
\hline \multirow[b]{2}{*}{ Variable } & \multicolumn{3}{|c|}{ Early pregnancy } & \multicolumn{3}{|c|}{ Middle pregnancy } \\
\hline & $\begin{array}{c}\text { Controls } \\
(n=156)\end{array}$ & $\begin{array}{c}G D M \\
(n=65)\end{array}$ & $p$ & $\begin{array}{l}\text { Controls } \\
(n=156)\end{array}$ & $\begin{array}{c}\text { GDM } \\
(n=65)\end{array}$ & $p$ \\
\hline Age (years) & $28 \pm 5$ & $31 \pm 5$ & & & & \\
\hline Weight (kg) & $65.1 \pm 11.9$ & $71.6 \pm 14.5$ & 0.0007 & $70.5 \pm 12.0$ & $77.4 \pm 14.2$ & 0.0003 \\
\hline BMI $\left(\mathrm{kg} / \mathrm{m}^{2}\right)$ & $25.2 \pm 4.3$ & $28.2 \pm 5.4$ & $<0.0001$ & $27.3 \pm 4.3$ & $30.5 \pm 5.2$ & $<0.0001$ \\
\hline Weight gain $(\mathrm{kg})$ & & & & $5.4 \pm 3.3$ & $5.8 \pm 4.7$ & ns \\
\hline Glucose (mg/dL) & $83.3 \pm 6.8$ & $88.8 \pm 8.5$ & $<0.0001$ & & & \\
\hline Insulin. $(\mu \mathrm{U} / \mathrm{mL})$ & $7.2 \pm 3.6$ & $9.9 \pm 5.6$ & $<0.0001$ & & & \\
\hline $\mathrm{HbA} 1 \mathrm{c}(\%)$ & $5.1 \pm 0.2$ & $5.4 \pm 0.3$ & & $4.9 \pm 0.3$ & $5.2 \pm 0.3$ & \\
\hline HOMA-IR & $1.4 \pm 0.8$ & $2.2 \pm 1.3$ & & & & \\
\hline OGTT mean glucose (mg/dL) & & & & $102.6 \pm 15.6$ & $128.3 \pm 26.3$ & \\
\hline $\operatorname{Irisin}(\mu \mathrm{g} / \mathrm{mL})$ & $0.8 \pm 0.5$ & $0.6 \pm 0.3$ & 0.01 & $1.3 \pm 0.7$ & $1.2 \pm 0.8$ & $\mathrm{~ns}$ \\
\hline
\end{tabular}

Data listed as mean $\pm S D$. A value of $p<0.01$ was considered statistically significant.

Table 2. Demographic and biochemical parameters and irisin levels of all pregnant women

\begin{tabular}{|c|c|c|}
\hline Variable & $\begin{array}{l}\text { Early pregnancy } \\
(n=221)\end{array}$ & $\begin{array}{l}\text { Middle pregnancy } \\
(\mathrm{n}=221)\end{array}$ \\
\hline Age (years) & $29 \pm 5$ & $29 \pm 5$ \\
\hline Weight (kg) & $67.02 \pm 13.11$ & $72.56 \pm 13.05$ \\
\hline $\mathrm{BMI}\left(\mathrm{kg} / \mathrm{m}^{2}\right)$ & $26.16 \pm 4.86$ & $28.32 \pm 4.8$ \\
\hline Weight gain(kg) & - & $5.5 \pm 3.81$ \\
\hline Glucose (mg/dL) & $84.98 \pm 7.81$ & - \\
\hline Insulin $(\mu \mathrm{U} / \mathrm{mL})$ & $8.01 \pm 4.5$ & - \\
\hline $\mathrm{HbA} 1 \mathrm{c}(\%)$ & $5.2 \pm 0.32$ & $5.07 \pm 0.36$ \\
\hline HOMA-IR & $1.7 \pm 1.03$ & - \\
\hline OGTT mean glucose (mg/dL) & & $110.2 \pm 22.6$ \\
\hline Irisin $(\mu \mathrm{g} / \mathrm{mL})$ & $0.75 \pm 0.54$ & $1.32 \pm 0.76$ \\
\hline
\end{tabular}

Data listed as mean $\pm S D$. A value of $p<0.1$ was considered statistically significant. 


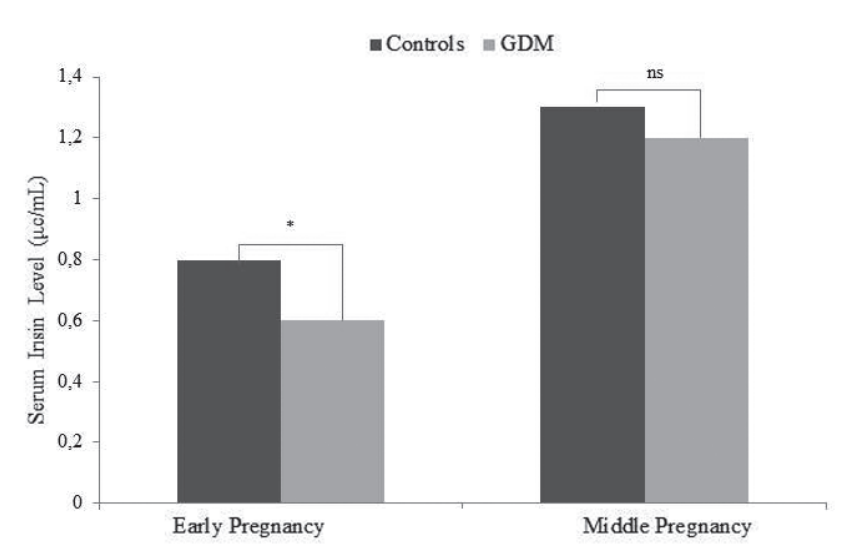

Figure 1. Difference of serum irisin levels in patients with GDM and healty controls during early and middle pregnancy. Serum irisin level was significantly lower in women with GDM during early pregnancy but the change was similar at middle pregnancy. $\left({ }^{*} \mathrm{p}=0.01\right)$.

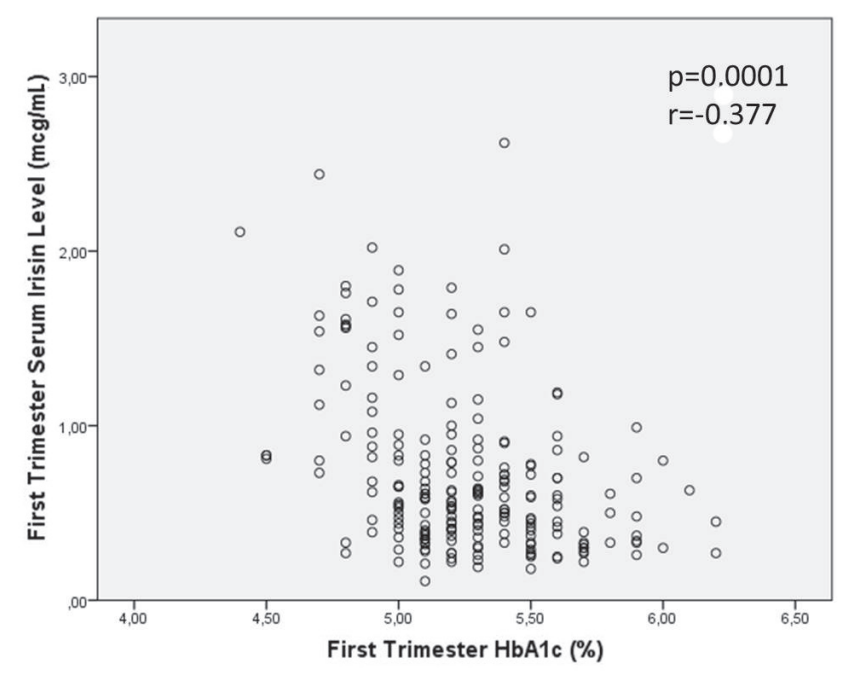

Figure 3. First trimester serum irisin level was negatively correlated with first trimester $\mathrm{HbA} 1 \mathrm{C}$.

$\mathrm{mL}$, respectively, $\mathrm{p}=0.01$ ) but no significant difference in middle pregnancy $(1.2847 \pm 0.893 \mu \mathrm{g} / \mathrm{mL} v s$, $1.34 \pm 0.705 \mu \mathrm{g} / \mathrm{mL}$ respectively, $\mathrm{p}=\mathrm{ns}$ ).

Correlations between serum irisin levels and metabolic parameters during normal pregnancy

In the univariate correlation analysis irisin serum levels negatively correlated with age in the early pregnancy $(r=-0.180, p=0.007)$, whereas no significant correlations are found in the middle pregnancy.

Analysis of pregnant women revealed that there was a significant inverse correlation of BMI with serum irisin in the early pregnancy $(\mathrm{r}=-0.193, \mathrm{p}=$ 0.004) (Fig. 2).

In addition, there was a significant inverse

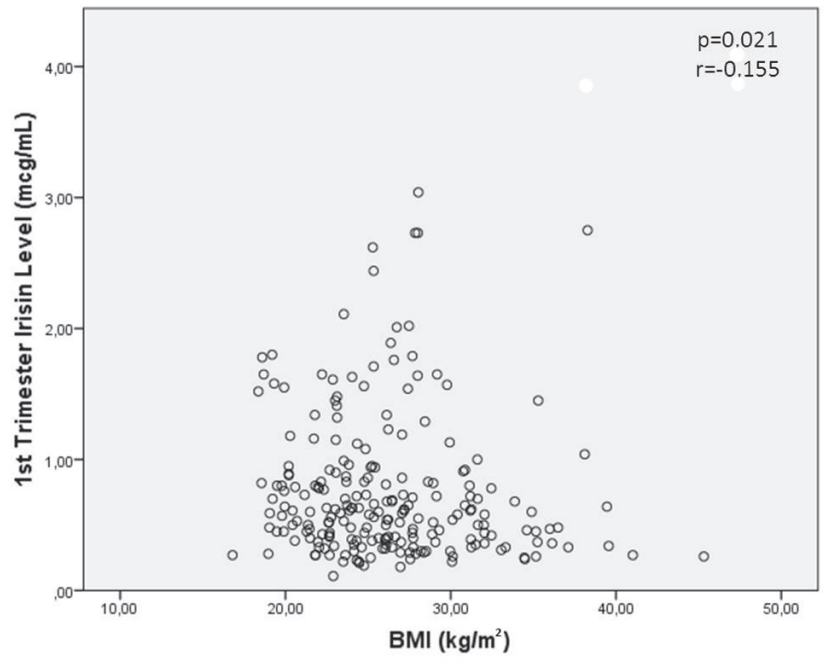

Figure 2. First trimester serum irisin level was negatively correlated with the body mass index.

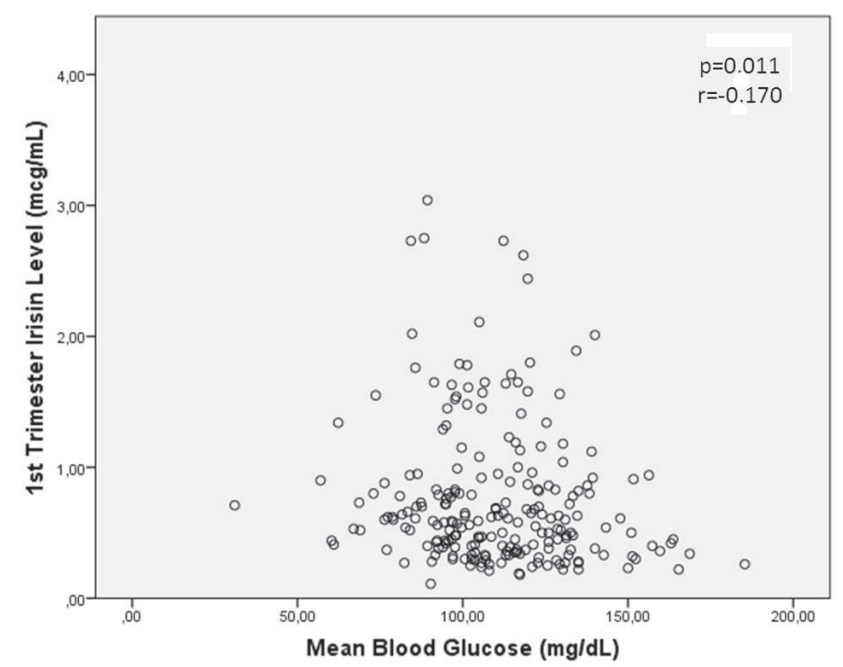

Figure 4. First trimester serum irisin level was negatively correlated with OGTT mean glucose level.

correlation of HbA1c and mean glucose of OGTT with serum irisin in the early pregnancy $(r=-0.377, p=0.0001$ (Fig. 3) and ( $r=-0.147$, p:0.03) (Fig. 4) respectively.

There was not any correlation between any parameters and the levels of irisin in the middle pregnancy.

\section{DISCUSSION}

Irisin has been proposed to be involved in mediating the beneficial effects of exercise on the metabolism and increase in levels of irisin induces browning of subcutaneous adipocytes and thermogenesis (3). Boström and co-workers showed that irisin overexpression results in improved glucose tolerance and increased energy expenditure in mice 
as compared to control animals (3). It was shown that irisin, a myokine potentially induced by acute exercise, thus plays an important role in obesity and metabolic disorders $(2,3,22)$.

The relationship between irisin and type 2 diabetes has been evaluated in recent studies $(4,13)$. It has been suggested that lower levels of circulating irisin are associated with diabetes itself or the metabolic conditions that cause progression to type 2 diabetes.

The anabolic condition of pregnant women contributes to positive energy balance and a significant increase in the accumulation of adipose tissue. It might be the cause of the GDM which is a prediabetic state or a transient unmasking of the metabolic syndrome and characterized by insulin resistance (7). Therefore, it can be speculated that irisin levels should be lower during gestation and decreased levels of irisin might be indicative of GDM.

However, the present study shows that irisin levels significantly increase with advancing gestation and serum irisin levels are significantly higher in the middle pregnancy compared with early pregnancy levels, with an increase in levels of about $61 \%$ in pregnant women. In subgroup analysis, serum irisin level increased about $60 \%$ throughout the gestational period from early to middle pregnancy in normal healthy pregnant women and $51 \%$ in pregnant women with GDM.

Our results confirm the findings of Garces et al., who also reported an increase in serum irisin with advancing gestation in pregnant women and additionaly found that irisin levels are higher in middle and late pregnancy with respect to early pregnancy (19). Kuzmicki et al. and Ebert et al., confirming this finding, also reported that serum irisin concentration increases markedly in pregnant women $(15,18)$.

The specific immunostaining of placental irisin precursor, FNDC5, suggests that the placenta might contribute to the increase in irisin serum levels during normal pregnancy (19).

When all these findings gathered together it can be speculated that as the fetus grow thoroughout the pregnancy energy requirement also increases. Irisin may function at this point to replace energy demand. This might be the reason of increased blood irisin level during gestational period.

On the other hand, it should also be expected to find a decrease in circulating irisin levels after the pregnancy. This is supported by the findings of Kuzmicki et al. and Ebert et al. who showed a decrease in irisin concentrations at the postpartum period compared to pregnancy levels $(15,18)$. But further studies are needed to establish these findings.

The authors suggested that the high irisin levels in pregnant women are a compensatory response caused by an irisin resistance, similar to leptin resistance seen in patients with obesity and/or metabolic syndrome (22-24) therefore resulting in decreasing browning and thermogenesis and the gain of fat.

Hee Park et al. proposed the concept of irisin resistance with compensatory hyperirisinemia and showed that high irisin levels were associated with an increased risk of the metabolic syndrome and cardiovascular disease (25).

These results are consistent with Liu and co-workers, report that circulating irisin was significantly lower in patients with type 2 diabetes, and that in non-diabetic individuals circulating irisin correlated positively with age, BMI, total cholesterol, triglycerides, fasting blood glucose and diastolic blood pressure (4). The positive association of circulating irisin with BMI was also reported in two non-diabetic populations, recently $(2,14)$. In these studies, morbidly obese patients had higher circulating levels of irisin than normal weight and anorexic patients (14), and weight-loss induced by bariatric surgery decreased both circulating irisin and muscle FDNC5 expression (2). The results provided by Liu and co-workers show that irisin may correlate positively with the most known markers of insulin resistance in non-diabetic population (4). As proposed previously, this could indicate that increased circulating irisin is an adaptive response to compensate for the decreasing insulin sensitivity (2).

Garcés et al. found that irisin levels were positively correlated with HOMA-IR in the univariate analysis and irisin may have a contributing role in the increase of insulin resistance in the normal pregnant women, regardless of the trimester of gestation and other variables (19).

There are studies which are supporting this finding: Kuzmicki and Ebert et al. reported that irisin levels were significantly lower in postpartum as compared to irisin concentrations during pregnancy $(15,18)$ which may suggest a compensation for a physiologic increase in insulin resistance.

Further analysis revealed that after adjusting for BMI, lipids and glucose, irisin levels were significantly lower in non-obese pregnant women as compared with obese and GDM women and serum irisin was positively correlated with fasting glucose, insulin and HOMA-IR (17).

In contrast, Kuzmicki et al. (only in the 
subgroup with non-diabetic women) and Yuksel et al. (in all pregnant women) reported that serum irisin level was negatively correlated with HOMA-IR $(15,16)$. Studies in obese patients found controversial results, with circulating irisin being positively associated with insulin sensitivity (26). However, an association between irisin and insulin resistance, in particular during pregnancy, seems still controversial.

Our results showed that fasting glucose and insulin levels and HOMA-IR are significantly higher in pregnant women with GDM than healthy pregnant women in early pregnancy, but there was no relationship between HOMA-IR and irisin. On the other hand, we did not find significant difference in circulating irisin in gestational diabetic pregnant women as compared with healthy pregnant women in the middle pregnancy.

Similar to our findings, Ebert et al. found no difference in circulating irisin between pregnant women with and without GDM in the middle pregnancy (18). Recently, Piya et al. has reported no significant differences in serum irisin between non-obese, obese and GDM women at term (17).

In contrast, Kuzmicki et al. reported that circulating irisin levels is significantly lower in women with GDM than in healthy pregnant women in the middle pregnancy (15). Similar to this findings, Yüksel et al. also reported that circulating irisin levels are significantly lower in women with GDM than in the healthy pregnant women; however, in this study irisin levels were measured at term (16).

On the other hand, we found significant difference in circulating irisin in gestational diabetic pregnant women as compared with healthy pregnant women in the early pregnancy. The studies which analyzed serum irisin levels, were in middle pregnancy in women with $\operatorname{GDM}(15,16,18)$. This is the first study determining plasma irisin levels in early pregnancy.

All these discrepancies may result from differences in clinical characteristics of the subjects studied and various diagnostic criteria; however, the possible influence of BMI or weight gain during pregnancy and gestational week at sampling seems unclear.

In the present study the women with GDM had significantly higher BMI than healthy pregnant women in early and middle pregnancy (p: 0.0007) and (p:0.0003) respectively. There is a significant inverse correlation of BMI with serum irisin of pregnant women in the early pregnancy $(r=-0.193, p=0.004)$.

In addition, there is no significant difference with gestational diabetic pregnant women as compared with healthy pregnant women in the amount of weight gained during pregnancy.

There is a positive correlation between serum irisin and BMI in the middle pregnancy (18) and a negative one at term (17). On the other hand, no association between circulating irisin and BMI or gestational age was observed (15).

Our results show that irisin increases during the gestation and irisin levels are significantly higher in middle pregnancy than in early pregnancy in total group and subgroups (healthy and GDM women). We can speculate that there is no significant difference of gestational diabetic pregnant women as compared with healthy pregnant women in the amount of weight gained during pregnancy and this may be the result of finding no difference between groups.

In conclusion, the present study shows: a) for the first time that serum irisin level increases throughout the gestational period from early to middle pregnancy in women with GDM; b) serum irisin levels rise during normal pregnancy; c) irisin cannot be used as a prospective and novel marker for GDM.

Further experiments are needed to fully explore the association between this new myokine/adipokine and glucose metabolism disturbances in pregnancy.

\section{Conflict of interest}

The authors declare that they have no conflict of interest.

\section{Acknowledgment}

This research received no grant from any funding agency in the public, commercial or not-for-profit sectors.

\section{References}

1. Boström PA, Graham EL, Georgiadi A, Ma X. Impact of exercise on muscle and nonmuscle organs. IUBMB Life 2013; 65(10): 845850 .

2. Huh JY, Panagiotou G, Mougios V, Brinkoetter M, Vamvini MT, Schneider BE, Mantzoros CS. FNDC5 and irisin in humans: I. Predictors of circulating concentrations in serum and plasma and II. mRNA expression and circulating concentrations in response to weight loss and exercise. Metabolism 2012; 61(12): 1725-1738.

3. Boström P, Wu J, Jedrychowski MP, Korde A, Ye L, Lo JC, Rasbach KA, Boström EA, Choi JH, Long JZ, Kajimura S, Zingaretti MC, Vind BF, Tu H, Cinti S, Højlund K, Gygi SP, Spiegelman BM. A PGC1- $\alpha$-dependent myokine that drives brownfat-like development of white fat and thermogenesis. Nature 2012; 481(7382):463-468.

4. Liu JJ, Wong MD, Toy WC, Tan CS, Liu S, Ng XW, Tavintharan $\mathrm{S}$, Sum CF, Lim SC. Lower circulating irisin is associated with type 2 diabetes mellitus. J Diabetes Complications 2013; 27(4): 365369.

5. Højlund K, Boström P. Irisin in obesity and type 2 diabetes. J Diabetes Complications 2013; 27(4): 303-304. 
6. Standards of medical care in diabetes-American Diabetes Association. Diabetes Care 2015; 38:Supplement 1:S8-S16.

7. Buchanan TA, Xiang A, Kjos SL, Watanabe R. What is gestational diabetes? Diabetes Care 2007; 30 Suppl 2:S105-S111.

8. Fadl HE, Ostlund IKM, Magnuson AF, Hanson USB. Maternal and neonatal outcomes and time trends of gestational diabetes mellitus in Sweden from 1991 to 2003. Diabet Med 2010; 27: 436441.

9. Kim C, Newton KM, Knopp RH. Gestational diabetes and the incidence of type 2 diabetes: a systematic review. Diabetes Care 2002; 25: 1862-1868.

10. Shah BR, Retnakaran R, Booth GL. Increased risk of cardiovascular disease in young women following gestational diabetes mellitus. Diabetes Care 2008; 31: 1668-1669.

11. Catalano PM, Kirwan JP, Haugel-de Mouzon S, King J. Gestational diabetes and insulin resistance: role in short- and long-term implications for mother and fetus. J Nutr 2003; 133: 1674S-1683S.

12. Fasshauer M, Blüher M, Stumvoll M. Adipokines in gestational diabetes. Lancet Diabetes Endocrinol 2014; 2: 488-499.

13. Choi YK, Kim MK, Bae KH, Seo HA, Jeong JY, Lee WK, Kim JG, Lee IK, Park KG. Serum irisin levels in new-onset type 2 diabetes. Diabetes Res Clin Pract 2013; 100: 96-101.

14. Stengel A, Hofmann T, Goebel-Stengel M, Elbelt U, Kobelt P, Klapp BF. Circulating levels of irisin in patients with anorexia nervosa and different stages of obesity-correlation with body mass index. Peptides 2013; 39: 125-130.

15. Kuzmicki M, Telejko B, Lipinska D, Pliszka J, Szamatowicz M, Wilk J, Zbucka-Kretowska M, Laudanski P, Kretowski A, Gorska M, Szamatowicz J. Serum irisin concentration in women with gestational diabetes. Gynecol Endocrinol 2014; 30: 36-39.

16. Yuksel MA, Oncul M, Tuten A, Imamoglu M, Acikgoz AS, Kucur M, Madazli R. Maternal serum and fetal cord blood irisin levels in gestational diabetes mellitus. Diabetes Res Clin Pract 2014; 104:171-175.

17. Piya MK, Harte AL, Sivakumar K, Tripathi G, Voyias PD, James S, Sabico S, Al-Daghri NM, Saravanan P, Barber TM, Kumar S, Vatish M, McTernan PG. The identification of irisin in human cerebrospinal fluid: influence of adiposity, metabolic markers, and gestational diabetes. Am J Physiol Endocrinol Metab 2014; 306:E512-E518.
18. Ebert T, Stepan H, Schrey S, Kralisch S, Hindricks J, Hopf L, Platz M, Lossner U, Jessnitzer B, Drewlo S, Blüher M, Stumvoll M, Fasshauer M. Serum levels of irisin in gestational diabetes mellitus during pregnancy and after delivery. Cytokine 2014; 65: 153-158. 19. Garcés MF, Peralta JJ, Ruiz-Linares CE, Lozano AR, Poveda NE, Torres-Sierra AL, Eslava-Schmalbach JH, Alzate JP, Sánchez AY, Sanchez E, Angel-Müller E, Ruíz-Parra AI, Diéguez C, Nogueiras R, Caminos JE. Irisin levels during pregnancy and changes associated with the development of preeclampsia. J Clin Endocrinol Metab 2014; 99: 2113-2119.

20. Alberti KG, Zimmet PZ. Diabet Med. Definition, diagnosis and classification of diabetes mellitus and its complications. Part 1: diagnosis and classification of diabetes mellitus provisional report of a WHO consultation. Diabet Med.1988; 15: 539-553.

21. Matthews DR, Hosker JP, Rudenski AS, Naylor BA, Treacher DF, Turner RC. Homeostasis model assessment: insulin resistance and P-cell function from fasting plasma glucose and insulin concentrations in man. Diabetologia 1985; 28: 412-419.

22. Anastasilakis AD, Polyzos SA, Saridakis ZG, Kynigopoulos G, Skouvaklidou EC, Molyvas D, Vasiloglou MF, Apostolou A, Karagiozoglou-Lampoudi T, Siopi A, Mougios V, Chatzistavridis P, Panagiotou G, Filippaios A, Delaroudis S, Mantzoros CS. Circulating irisin in healthy, young individuals: day-night rhythm, effects of food intake and exercise, and associations with gender, physical activity, diet, and body composition. J Clin Endocrinol Metab. 2014; 99(9):3247-3255.

23. Zhang Y, Scarpace PJ. The role of leptin in leptin resistance and obesity. Physiol Behav 2006; 88: 249-256.

24. Henson MC, Castracane VD. Leptin in pregnancy: an update. Biol Reprod 2006; 74: 218-229.

25. Lain KY, Catalano PM. Metabolic changes in pregnancy. Clin Obstet Gynecol 2007; 50: 938-948.

26. Park KH, Zaichenko L, Brinkoetter M, Thakkar B, Sahin-Efe A, Joung KE, Tsoukas MA, Geladari EV, Huh JY, Dincer F, Davis CR, Crowell JA, Mantzoros CS. Circulating irisin in relation to insulin resistance and the metabolic syndrome. J Clin Endocrinol Metab 2013; 98: 4899-4907.

27. Moreno-Navarrete JM, Ortega F, Serrano M, Guerra E, Pardo G, Tinahones F, Ricart W, Fernández-Real JM. Irisin is expressed and produced by human muscle and adipose tissue in association with obesity and insulin resistance. J Clin Endocrinol Metab 2013; 98: E769-E778. 